\title{
AUTONOMIA E CRIATIVIDADE NO TRABALHO DE EQUIPES DE SAÚDE DA FAMÍLIA NO SUL DO BRASIL
}

\author{
Autonomy And Creativity In The Work Of Health Teams \\ Of The Family In The South Of Brazil \\ Autonomie Et Créativité Dans Le Travail De L'équipe De Santé \\ Famille Dans Le Sud Brésil \\ Autonomía Y Creatividad En El Trabajo De Equipos De Salud \\ La Familia En El Sur De Brasil
}

Cecília Helena Glanzner ${ }^{1}$

Professora da Universidade Federal do Rio Grande do Sul, Departamento de Enfermagem Médico Cirúrgica.

Pesquisadora do Grupo Interdisciplinar de Saúde Ocupacional. Possui Graduação em Enfermagem (2000),

Licenciatura em Enfermagem (2001), Mestrado em Enfermagem pela Universidade Federal do Rio Grande do

Sul (2008). Doutorado em Enfermagem pela Universidade Federal do Rio Grande do Sul (2014). Trabalhou

como enfermeira do Hospital de Clínicas de Porto Alegre, Hospital Nossa Senhora da Conceição S/A e como

Professora do Instituto de Ciências da Saúde da Universidade Feevale. Tem experiência na área de Enfermagem cirúrgica e Bloco Cirúrgico e, em pesquisas comênfase na área de saúde, saúde mental do trabalhador e avaliação de serviços de saúde.

Agnes Olschowsky ${ }^{2}$

Doutorado em Enfermagem Psiquiátrica pela Escola de Enfermagem da USP (2001), mestrado em Enfermagem Psiquiátrica pela Universidade de São Paulo (1996) e graduação em Enfermagem e Obstetrícia pela Universidade do Vale do Rio dos Sinos (1982). Atualmente é Professora Titular da Universidade Federal do Rio Grande do Sul, docente credenciado junto ao Programa de Pós-Graduação em Enfermagem da UFRGS, membro de Conselho da Associação Brasileira de Enfermagem e membro do Conselho Editorial da Revista Gaúcha de

Enfermagem. Coordenadora da pesquis a valiação das ações de saúde mental na ESF, financiada pelo CNPq e MS. Tem experiência na área de Enfermagem, com ênfase em Enfermagem Psiquiátrica, atuando principalmente nos seguintes temas: enfermagem, saúde mental, enfermagem psiquiátrica, ensino de enfermagem, prazer e sofrimento no trabalho e ações de saúde mental na atenção básica.

Deisi Angélica Hoffmann ${ }^{3}$

Graduanda de Enfermagem da Escola de Enfermagem da Universidade Federal do Rio Grande do Sul - UFRGS

\section{RESUMO}

A Saúde da Família tem um espaço muito importante em todo território brasileiro e é fundamental para os cuidados em saúde da população. Os profissionais inseridos nesse meio lidam diariamente com situações novas e imprevistas ligadas às demandas dos usuários devendo ser dotados de conhecimento para atendê-los de forma

\footnotetext{
${ }^{1}$ E-mail: glanznercecilia@gmail.com

2 E-mail: agnes@portoweb.com.br

${ }^{3}$ E-mail: deisi_hoffmann@hotmail.com
} 
resolutiva. Nesse sentido, a autonomia e criatividade profissional desempenham um papel importante, pois quando presente relaciona-se à estabilidade psíquica dos profissionais, sendo um fator de prazer no ambiente de trabalho. O objetivo do artigo é analisar a autonomia e criatividade no trabalho dos profissionais de saúde da família do sul do Brasil. Trata-se de um estudo com abordagem qualitativa que teve como referencial teórico a psicodinâmica do trabalho e foi realizado com profissionais de equipes de saúde da família de três Unidades de Saúde pertencentes a um grupo hospitalar no sul do Brasil. Para coleta das informações foram utilizadas a observação e entrevistas coletivas. Para a análise dos dados foi utilizada o método de análise de conteúdo. Os profissionais das equipes de Saúde da Família consideram a autonomia e criatividade como fonte de prazer no trabalho, pois através dela foge se da robotização no trabalho, possibilita a personalização nas tarefas em que o profissional tem liberdade de demonstrar seus conhecimentos, experiências e capacidades para organizar e produzir seu trabalho. Conclui-se que os profissionais das equipes investigadas se referem ao seu trabalho como prazeroso, identificam sentimentos de satisfação no trabalho quando realizado com autonomia e criatividade. Por outro lado, quando não existe diálogo entre o trabalho prescrito e o profiss ional com seu potencial subjetivo e criativo na construção do trabalho, convive com o sofrimento.

Palavras-chaves: Autonomia Profissional, Criatividade, Saúde da Família, Avaliação em Saúde.

\section{ABSTRACT}

Family Health has a very important space throughout Brazil and is fundamental for the health care of the population. The professionals inserted in this environment, deal daily with new unforeseen situations linked to the demands of the users and must be endowed with the knowledge to serve them in a resolutive way. In this sense, professional autonomy and creativity play an important role, because when present it is related to the psychic stability of professionals, being a pleasure factor in the work environment. The objective of the article is to analyze the autonomy and creativity in the work of the health professionals of the family of the south of Brazil. It is a study with a qualitative approach that had as theoretical reference the psychodynamics of the work and was realized with professionals of health teams of the family of three Health Units belonging to a hospital group in the south of Brazil. Data collection was used to observe and collective interviews. For data analysis was used the content analysis method. The professionals of the Family Health teams consider autonomy and creativity as a source of pleasure at work, because through it, one steers away from robotization at work, allows personalization in tasks in which the professional is free to demonstrate their knowledge, experiences and abilities to organize and produce their work. It is concluded that the professionals of the investigated teams refer to their work as pleasurable, they identify feelings of satisfaction at work when performed with autonomy and creativity. On the other hand, when there is no dialogue between prescribed work and professional with its subjective and creative potential in the construction of work, it coexists with suffering.

Keywords: Professional Autonomy, Creativity, Family Health, Evaluation In Health.

\section{RÉSUMÉ}

La santé de la famille a une place très importante au Brésil et est fondamental pour la prise en charge de la santé. Les professionnels impliqués dans l'affaire quotidienne moyenne avec une nouvelle situations imprévue liée aux demandes des utilisateurs devraient être des connaissances pour les aider à résoudre manière. En ce sens, l'autonomie et la créativité professionnelle jouent un rôle important, parce que quand cela est lié à la stabilité mentale des professionnels, étant un facteur de plaisir dans le lieu de travail. Le but de cet article est d'analyser l'autonomie et la créativité dans le travail des professionnels de la santé dans la famille du sud du Brésil. Ceci est une étude qualitative qui avait comme référence théorique la psychodynamique du travail et a été réalisée avec des professionnels de trois équipes de santé familiale Unités de santé appartenant à un groupe hospitalier dans le sud du Brésil. Pour l'observation collection ont été utilisées des données et conférences de presse et analyse des données a été utilisé la méthode d'analyse du contenu. Les professionnels des équipes de santé de la famille considèrent l'autonomie et la créativité comme source de plaisir au travail, car à travers elle on fuit devant le robot au travail, permet la personnalisation des tâches dans lesquelles le professionnel est libre de démontrer leurs connaissances, l'expérience et les capacités de organiser et produire leur travail. Il est conclu que les équipes professionnelles étudiées se réfèrent à son travail ag réable, identifier les sentiments de satisfaction au travail lorsqu'elle est effectuée avec l'autonomie et la créativité. D'autre part, quand il n'y a pas de dialogue entre le travail prescrit et professionnel avec leur potentiel subjectif et créatif dans les travaux de construction, vivant avec la souffrance.

Mots-Clés: Autonomie Professionnelle, Créativité, Santé Familiale, Évaluation Santé. 


\section{RESUMEN}

La Salud de la Familia tiene un espacio muy importante en todo el territorio brasileño y es fundamental para los cuidados en salud de la población. Los profesionales insertados en ese medio tratan diariamente con situaciones nuevas y imprevistas ligadas a las demandas de los usuarios debiendo ser dotados de conocimiento para atenderlos de forma resolutiva. En ese sentido, la autonomía y la creatividad profesional desempeñan un papel importante, pues cuando presente se relaciona con la estabilidad psíquica de los profesionales, siendo un factor de placer en el ambiente de trabajo. El objetivo del artículo es analizar la autonomía y la creatividad en el trabajo de los profesionales de salud de la familia del surde Brasil. Se trata de un estudio con abordaje cualitativo que tuvo como referencial teórico la psicodinámica del trabajo y fue realizado con profesionales de equipos de salud de la familia de tres Unidades de Salud pertenecientes a un grupo hospitalario en el sur de Brasil. Para la recolección de las informaciones fueron utilizadas la observación y entrevistas colectivas y el análisis de los datos fue utilizado el método análisis de contenido. Los profesionales de los equipos de Salud de la Familia consideran la autonomía y la creatividad como fuente de placer en el trabajo, pues a través de ella se escapa de la robotización en el trabajo, posibilita la personalización en las tareas en que el profesional tiene libertad de demostrar sus conocimientos, experiencias y capacidades para organizar y producir su trabajo. Se concluye que los profesionales de los equipos investigados se refieren a su trabajo como placer, identifican sentimientos de satisfacción en el trabajo cuando se realiza con autonomía y creatividad. Por otro lado, cuando no existe diálogo entre el trabajo prescrito y el profesional con su potencial subjetivo y creativo en la construcción del trabajo, convive con el sufrimiento.

Palabras Clave: Autonomía Profesional, Creatividad, Salud de la Familia, Evaluación En Salud.

\section{INTRODUÇÃO}

O surgimento da PDT (Psicodinâmica do Trabalho) ocorreu na década de 1980 com o médico francês Christophe Dejours que iniciou a discussão sobre o sofrimento psíquico do trabalhador e as estratégias de enfrentamento diante da atividade laboral (Dejours, 2004a). Desde lá, o interesse ao conhecimento dos fatores ligados a esse tema tem aumentado em todo mundo sendo alvo de pesquisas, debates e exposições em congressos (Bueno \& Macêdo, 2012).

A PDT, tem como objetivo enfatizar o estudo das relações dinâmicas entre a organização do trabalho e os processos subjetivos e intersubjetivos que surgem da relação entre a organização psíquica das pessoas com o processo laboral. Está intrinsecamente ligada a fala coletiva dos trabalhadores, a compreensão da dimensão constitutiva e positiva do trabalho, as vivências de prazer e sofrimento bem como sua relação com o processo saúdeadoecimento no ambiente de trabalho vendo o trabalhador como sujeito complexo que não se limita apenas ao seu comportamento (Dejours, 1992).

$\mathrm{O}$ prazer surge como sentimento profundamente benéfico ligado à estabilidade psíquica do trabalhador quando passa a superar os desafios impostos pelo trabalho, pois percebe-se com habilidades adquiridas e dotado da resolutividade em seu meio laboral (Moraes, Vasconcelos \& Cunha, 2012). Já o sofrimento é dado com uma experiência emocional desagradável associado a um conjunto de sentimentos como o medo, desvalia, impotência, insatisfação, estresse entre outros. As condições e prescrição do trabalho associadas a característica do indivíduo podem resultar no surgimento do sofrimento (Dejours, 2008).

A tarefa prescrita é definida como as condições e exigências impostas para que o trabalho seja efetivado, ela leva em consideração o ambiente físico e a matéria prima, as regras, ordens e metas para atingir os resultados propostos. Já na tarefa real, considera-se todas as questões intrínsecas do trabalhador que precisam ser acionadas para a realização da tarefa como a experiência, lidar com os imprevistos, reconhecer falhas no processo, adaptação de tarefas entre outros (Silva \& Ramminger, 2014). De acordo com Dejours (2008) toda atividade não prescrita exige inventividade, criatividade e formas de inteligência específicas denominada como "engenhosidade" ou "inteligência da prática do corpo". 
E nesse contexto, se insere o trabalho na SF (Saúde da Família) que consiste inicialmente em compreender a existência de um sujeito que vive em um território, estabelece relações sociais, faz parte de uma família e tem uma necessidade objetiva ou subjetiva de saúde que o faz buscar o atendimento. Isso exige um processo de interação e relação entre os sujeitos envolvidos para a produção de saúde, portanto, é desempenhado de uma forma singular pelos profissionais (Lucchese et al., 2014).

Nesse cenário e frente à sua dimensão, o trabalho dos profissionais das equipes de SF se caracteriza como criativo, possibilitando ao trabalhador organizá-lo de diferentes maneiras, possibilitando $\mathrm{o}$ exercício da autonomia profissional em suas atividades laborais. Isso pode ser vis to como duplamente positivo, pois $\mathrm{O}$ profissional sente-se valorizado uma vez que sua experiência é levada em consideração nas tomadas de decisões e o usuário recebe um atendimento voltado para sua verdadeira necessidade estabelecendo vínculo com a unidade de saúde (Glanzner, 2014).

Portanto, a autonomia está ligada a possibilidade de os humanos regerem suas próprias leis, ao invés de receberem de outros, refere-se a liberdade moral e intelectual para usar próprias regras que vão definir sua conduta. Considerada como a capacidade de reflexão e ação crítica, a autonomia foi considerada um ingrediente importante para impor limites ao poder de grupos dominantes (Campos et al., 2012). O homem autônomo é aquele considerado crítico, reflexivo, democrático e que se reconhece pertencente a uma sociedade (Castoriadis, 1987).

A criatividade envolve a capacidade de criar e inventar sendo considerado criativo pessoas que tem ideias originais. Quando há espaço para a mobilização da subjetividade no ambiente de trabalho e o exercício da criatividade, o trabalhador adquire autonomia, que contribui efetivamente com o processo de trabalho, com o desenvolvimento da sua estabilidade psíquica e construção da identidade no meio em que trabalha, sendo fonte de prazer (Dejours, 2008).

Por outro lado, quando o ambiente de trabalho é fechado e exige do trabalhador apenas a tarefa prescrita, não estimula mobilização subjetiva que impulsiona a autonomia e criatividade, não estabelecendo uma via de comunicação entre a tarefa real e seu executor, isso pode gerar sentimentos de insatisfação contribuindo para o sofrimento no trabalho (Dejours, 2008). Sendo assim, podemos afirmar que a autonomia e criatividade andam no mesmo sentido podendo influenciar de forma positiva ou negativa a saúde no trabalho.

O trabalho voltado para a exigência de alcançar objetivos e produção quantitativa impacta tanto na qualidade da assistência prestada como na condição de saúde do trabalhador. Estudos recentes mostram dados alarmantes referente a saúde dos trabalhadores em relação ao trabalho, no qual foram evidenciados sentimentos desvalia e inutilidade, dificuldade de desempenhar a atividade proposta, relacionando esses sentimentos a um prejuízo a vida e relações pessoais do trabalhador contribuindo para ocorrência de transtorno mental ou distúrbios psiquiátricos (Merlo, Bottega \& Perez, 2016).

Desse modo, diante do contexto de trabalho na SF, questiona-se se os profissionais realizam suas atividades laborais com autonomia e criatividade e se está relacionado ao prazer ou sofrimento no trabalho. Assim, o objetivo do artigo é analisar a autonomia e criatividade no trabalho dos profissionais de saúde da família do sul do Brasil.

\section{Metodologia}

Trata-se de um estudo com abordagem qualitativa que teve como referencial teórico a Psicodinâmica do Trabalho e foi realizado com profissio na is 
de equipes de Saúde da Familia de três Unidades de Saúde (US) no sul do Brasil.

Foram selecionadas três US que compõem um serviço com doze US pertencentes a um grupo hospitalar distribuídas em dois distritos sanitários da cidade. A escolha foi intencional, relacionada com os resultados de uma prépesquisa aplicada nas doze US e que obtiveram como resultado: alto, intermediário e baixo risco de adoecimento relacionado ao trabalho (Glanzner, 2014). Foram identificadas com as letras A, B e C.

Todos os participantes do estudo eram profissionais das equipes de SF que estavam trabalhando nas três US selecionadas no momento da coleta de informações. Na US A participaram 13 profissionais, 30 na US B e 25 na US C, totalizando 68 profissionais, dentre eles enfermeiros, técnicos de enfermagem, médicos, dentistas, psicólogos, técnicos em saúde bucal, assistente social.

As informações foram coletadas a partir de observação de campo e entrevis tas coletivas após aprovação do Comitê de Ética em Pesquisa da instituição e contato com a Coordenadora, Gerente da Saúde Comunitária e Coordenadores das US.

Foram realizadas vinte horas de observação em cada US, nos turnos manhã e tarde, totalizando sessenta horas de observação que foram registradas em um diário de campo. A coleta de informações foi realizada nos meses de outubro, novembro e dezembro de 2012.

Na PDT, a observação clínica é essencial e deve ser fundamentada nos discursos dos participantes da sessão. Seguindo os passos da PDT, deu-se continuidade ao estudo em que foram realizadas entrevistas coletivas com os sujeitos (Dejours, 2004b). O propósito da entrevista é permitir ao avaliador entrar na perspectiva do outro, buscando apreender sentimentos, pensamentos e intenções (Patton, 2002).

$\mathrm{Na}$ redação do texto, as entrevistas coletivas foram identificadas com as especificações EC para entrevista coletiva, acrescida do número conforme a US e, letras $\mathrm{A}, \mathrm{B}$ e $\mathrm{C}$ sucessivamente para diferenciar os profissionais, EC1-A, EC2-A e EC3-B.

As entrevistas coletivas com as equipes de SF foram divididas em dois momentos, primeiramente foi destinado a apresentação da pesquisa e convite para a entrevista coletiva e o segundo momento, foi destinado a realização da entrevista coletiva propriamente dita com cada uma das três equipes de SF. Desse modo, a entrevista coletiva permitiu que os profissionais entrevistados falassem livremente sobre o tema proposto, conforme as questões norteadoras da entrevista coletiva, como segue: fale sobre o seu trabalho na SF; fale sobre os fatores que causam prazer em seu trabalho; e fale sobre situações que the causam sofrimento no trabalho. Como lida com isso?

Os encontros tiveram duração de duas horas em cada US e após, iniciou-se a transcrição das entrevistas na íntegra e organização das anotações de campo. A análise das informações foi realizada utilizando-se o método de análise de conteúdo que foi constituída de três passos fundamentais: pré-análise, exploração do material, tratamento dos resultados obtidos e interpretação (Bardin, 2009).

Foram realizados os passos conforme os preceitos éticos da Resolução 466/2012, sendo a pesquisa aprovada pelo Comitê de Ética em Pesquisa da instituição sob parecer número 11-140. Os sujeitos foram convidados a participar da pesquisa e aqueles que concordaram assinaram o Termo de Consentimento Livre e Esclarecido (Ministério da Saúde, 2012).

\section{Resultados e discussão}

Nas discussões coletivas com os profissionais das equipes de SF no sul do Brasil, a autonomia e criatividade são consideradas como fonte de prazer no trabalho, proporcionando satisfação ao realizarem suas atividades profissionais. 
A noção de autonomia depende de condições culturais e sociais. Portanto, é necessário apreender diversos saberes e culturas para que seja possível decidir e refletir de forma autônoma sobre quais ideias seguir (Morin, 2011).

Para os profissionais entrevistados as regras rígidas do trabalho oferecem tranquilidade, e, ao mesmo tempo, ter autonomia para realizarem suas atividades, se associa a responsabilidade, produzindo satisfação no trabalho por imprimir sua marca pessoal.

Eu acho que uma das coisas boas que eu vejo nessa equipe, no trabalho, é a questão da autonomia, a gente tem autonomia, eu, pelo menos como profissional tenho autonomia (EC1C-3/10/12).

A autonomia é mais difícil de exercitar do que cumprir regras, então, essa coisa é contraditória. A gente gosta de autonomia, mas, ela é trabalhosa. E, às vezes por tabelinha, cria problemas de relacionamento. Mas, ela é boa porque deixa nas mãos $d a$ gente o processo $e$, não só obedecer às regras que tem. (EC2 -E - 3/12/12).

A autonomia, sob a ótica dos profissionais das equipes de $\mathrm{SF}$, é prazerosa, pois foge da robotização no trabalho, imprimindo características pessoais em determinado trabalho por meio da expressão da subjetividade, na qual o profissional tem liberdade de demonstrar seus conhecimentos e capacidade para organizar e produzir seu trabalho. Assim, a autonomia para propor novas formas de organizar o trabalho mostra-se importante ao favorecer o exercício da inteligência prática, possibilitando que o sujeito crie novas formas, mais eficazes, de desempenhar suas tarefas, ou seja, há um investimento subjetivo que se reveste de prazer, independente do esforço necessário para organizar o trabalho (Moraes, Vasconcelos \& Cunha, 2012).

Nesse sentido, a autonomia implica em responsabilidade, porque oferece liberdade com compromisso, ou seja, no cotidiano do trabalho a organização ocorre nas relações produzidas, exigindo dos profissionais, capacidades para enfrentar as condições do trabalho real, ou seja, aquele em que há conflitos, interesses diversos, em que o trabalho também é sofrimento e, por meio da mobilização subjetiva do sujeito, é transformado em prazer.

A autonomia no trabalho pode ser compreendida como a possibilidade de alteração da prescrição da sua tarefa de forma a adequá-la ao real do trabalho, possibilitando ao trabalhador a regulação de seu modo de desenvolver atividades (Ferreira, 2011). Assim, uma organização de trabalho flexível valoriza o exercício da inteligência prática, da criação e da invenção do novo, favorecendo o prazer, por meio do exercício da autonomia, com base na transformação do sofrimento em prazer de saber fazer (Moraes, Vasconcelos \& Cunha, 2012).

O estudo de Moraes, Vasconcelos \& Cunha (2012) relata que no cenário da SF, o enfermeiro encontra um importante espaço de atuação de trabalho que pode fortalecer a consolidação do SUS, se o contexto sóciohistórico e a ambiguidade das relações de poder na prática social da profissão for melhor compreendido, aperfeiçoando-o crítica, coletiva e criativamente.

Nesse sentido, a autonomia política, capaz de enfrentar as contradições da prática, articula-se intrinsecamente com a dimensão técnica do fazer, dando-lhe força argumentativa e coesão de sujeitos em torno de ideais comuns, o que evidencia a importância do exercício da autonomia na organização do trabalho, pois, ao se exercitar o poder de criar e dar características próprias ao fazer, isso se traduz em liberdade e, consequentemente, em estabilidade psíquica.

Portanto, percebe-se a necessidade de se desenvolver uma cultura crítica de 
reflexão sobre as fragilidades políticas das profissões da saúde para que a busca pela autonomia seja um exercício para qualific ar o fazer desses profissionais e o compromisso com as mudanças do modelo assistencial, permitindo imprimir a subjetividade do sujeito no trabalho e para que perceba sua atividade como algo prazeroso (Pires, 2011).

Desse modo, evidencia-se que os profissionais das equipes de SF devem desenvolver/utilizar estratégias para implementar o desenvolvimento da autonomia com poder para criar e inventar o seu trabalho, sentindo-se sujeitos do seu trabalho, e que o exercício da autonomia produza sentido ao seu trabalho e resulte em entusiasmo pelo que fazem.

O exercício da autonomia também se associa a criatividade no trabalho das equipes de SF. Assim, a criatividade gera a possibilidade de propor novas práticas, enfrentando o trabalho prescrito e rotineiro com a liberdade de criação e transformação daquilo que pode fazer o trabalhador sofrer.

[...] mas quando tu vê o grau de liberdade que tu tens aqui, no que já foi dito em relação a criar o teu trabalho, aí tu propõe novas práticas, isso dá uma possibilidade de um pouco mais de saúde no teu trabalho, coisa que eu não conseguiria num hospital [...] A questão da criação está na maioria dos processos todos, inclusive, quanto mais liberdade tiver "desplanejamento" se é que existe esta palavra? Melhor ainda [...] É disso que se trata, a equipe inteira ter uma noção do porque estamos fazendo isso. Bom, isso vai gerar muito sofrimento por uma questão até marxista alienada do teu trabalho, por uma visão de que esta criação precisa de planejamento. Aliás, tem que ser mais livre possível, mas você precisa saber porque está fazendo (EC1-C - 3/10/12).
Se alguém aqui faz trabalhos rotineiros, se sente, nessa coisa repetitiva que causa um certo tédio, uma possível fonte de desânimo, desinteresse é porque não está se permitindo inventar no trabalho porque existe essa possibilidade de tu, ser criativo no trabalho da atenção primária (EC1- E - 3/10/12).

Os profissionais sugerem $\mathrm{o}$ "desplanejamento", ou seja, saber o que se faz para ser livre e criar em seu trabalho; deixar de cumprir o rotineiro para exercer a liberdade de criação, propor novas práticas, contrapondo-se aos espaços de trabalho estruturados e engessados que impedem a possibilidade de criar. Criatividade e liberdade são percebidos como fontes de prazer na organização do seu trabalho. Ser criativo é interferir e agir na concepção do trabalho, ter conhecimento da sua finalidade, os meios e instrumentos necessários para sua construção e realização.

A liberdade também implica capacidade de reconhecer limites em si, nos outros, para si e para os outros. Agir livremente está associado a possibilidade de escolha e essa possibilidade está presente nas atividades humanas, e além do pensamento e criação ela se expressa quando se exerce a autonomia (Reibnitz \& Prado, 2003).

A questão da criatividade e liberdade no trabalho vem sendo discutida em amplo espectro, inclusive que deve iniciar na formação pedagógica como ferramenta para formação do profissional crítico-criativo. Nesse sentido, a valorização do espaço intercessor é de fundamental importância enquanto um privilegiado espaço de liberdade e autonomia, resgatando o potencial criativo e o potencial dos indivíduos (Reibnitz \& Prado, 2003).

O espaço intercessor é o encontro em que, cada uma das partes, usuário e trabalhador colocam suas necessidades, projetos, ansiedades, dores, medos, desejos, 
sonhos e potencialidades em dado contexto (Pires, 2011). Portanto, é nesse espaço do encontro entre trabalhador/usuário que se tem grande potência criativa, atravessado por determinantes externos (organizações, crenças, formação) que influenciam equipe e usuário, mas marcado por importante grau de liberdade e responsabilidade na ação desses profissionais no trabalho em saúde.

Assim, o espaço intercessor surge e passa a funcionar como um mediador para a saúde, pois pode transformar o sofrimento em criatividade, trazendo uma contribuição que beneficia a identidade e aumenta a resistência do trabalhador ao risco de desestabilização psíquica e somática (Merhy et al., 2007). E a criatividade pode ser mediadora de saúde por meio da mobilização subjetiva e proporcionar prazer, mas também pode desencadear a fragilização da saúde e gerar sofrimento ao profissional.

O trabalho é lugar de prazer quando é possível aprender sobre um fazer específico, criar, inovar e desenvolver novas formas para a execução da tarefa, quando são oferecidas condições de interagir com os outros, de socialização e reforço de uma identidade pessoal, e quando a organização do trabalho permite o estabelecimento de acordos que levem a transformação do sofrimento (Mendes, 2008).

Nesse sentido, a criatividade e prazer quando os profissionais das equipes das SF estudadas exercitam a liberdade de fazer, de inventar e sair da rotina prescrita do trabalho, exercendo sua autonomia, produzindo um "desplanejamento" e novas práticas, em que criar e saúde no trabalho. Por outro lado, a criatividade - enquanto inteligência prática que surge do enfrentamento do fracasso no trabalho, seja pelas relações positivas ou negativas e pelo modo de organização - é percebida como uma exigência constante no trabalho, caracterizando-o como fatigante, o que confere risco grave de adoecimento para o trabalho. Ou seja, a potência criativa do trabalho implica também, em cooperação coletiva, uma vez que o trabalho não é individual, mas se organiza na relação com outros.

\section{Conclusão}

No cotidiano laboral dos profissionais de equipes de $\mathrm{SF}$, a autonomia e criatividade estão presentes e, assim, permite dar voz às perspectivas desse trabalho com o intuito de mobilizar mudanças na organização do trabalho na busca do prazer.

A autonomia e a criatividade foram avaliadas como eventos de prazer no seu trabalho, pois produzem sensações agradáveis aos profissionais. A autonomia imprime características pessoais ao trabalho, potencializando a expressão da subjetividade, na qual o profissional tem liberdade de demonstrar seus conhecimentos e capacidade para organizar e produzir seu trabalho, enfrentando uma robotização das ações frente ao trabalho prescrito. A autonomia implica, também, responsabilidade porque oferece liberdade com compromisso, exigindo dos profissionais, capacidades para enfrentar as condições de trabalho e o trabalho real, permeado de conflitos e interesses diversos.

Nesse sentido, o exercício da autonomia também produz criatividade no trabalho das equipes de SF, propondo novas práticas e o enfrentamento do trabalho prescrito e rotineiro. Avaliam que ser criativo é interferir e agir na concepção do trabalho, ter conhecimento da sua finalidade, dos meios e instrumentos necessários para sua construção e realização.

Como forma de estimular a equipe para alcançar o prazer no ambiente de trabalho é necessário identificar situações causadoras de sofrimento no cotidiano dos profissionais, bem como buscar estratégias para a valorização profissional, propondo ambientes de conversa e estimulando a autonomia e criatividade no trabalho. 
Por meio da inteligência prática os profissionais são criativos e tem liberdade para inventar seu trabalho, o que mobiliza a cooperação coletiva uma vez que o trabalho não é exclusivamente individual e se organiza na relação com outros.

Assim conclui-se que os profissionais das equipes investigadas se referem ao seu trabalho como prazeroso, identificam sentimentos de satisfação no trabalho quando realizado com autonomia e criatividade. Por outro lado, quando o trabalhador não consegue negociar o trabalho prescrito com seu potencial subjetivo e criativo na construção do trabalho convive com o sofrimento.

\section{Referências}

Bardin, L. (2009). Análise de Conteúdo. Lisboa: Edições 70.

Bueno, M., \& Macêdo, K.B. (2012). A Clínica psicodinâmica do trabalho: de Dejours às pesquisas brasileiras. Estudos Contemporâneos da Subjetividade,2(2), 306-318. Retrieved in jun, 16, 2017 fromhttp://www.uff.br/periodicoshu manas/index.php/ecos/article/view/1 $\underline{010 / 723}$

Castoriadis, C. (1987). Encruzilhadas do Labirinto II 3/4 Domínios do Homem. (Tradução: José Oscar de Almeida Marques). Rio de Janeiro: Paz e Terra.

Campos, G.W.S., Carvalho, Y.M., Minayo, M.C.S., Drumond Junior, M., Akerman, M. (2012). Tratado de Saúde coletiva: Revista e Aumentada. ( $2^{\mathrm{a}}$ ed.) São Paulo: HUCITEC.

Dejours, C. (1992). Loucura do Trabalho: Estudo de Psicopatologia do Trabalho. São Paulo: Cortez.

Dejours C. (2004a). Christophe Dejours: da psicopatologia à psicodinâmica do trabalho. Rio de Janeiro: Fiocruz.
Dejours, C. (2004b). A metodologia em psicodinâmica do trabalho. Rio de Janeiro: Fiocruz.

Dejours, C. (2008). Inteligência prática e sabedoria: duas dimensões desconhecidas do trabalho real. $\left(2^{\mathrm{a}}\right.$ ed.) Rio de Janeiro: Fiocruz.

Ferreira M.C. (2011). Qualidade de Vida no Trabalho. Uma Abordagem Centrada no Olhar dos Trabalhadores/Mario Cesar Ferreira. Brasilia: Edições Ler, Pensar, Agir. Disponível em: http://ergopublic.com.br/arquivos/13 59392512.36-arquivo.pdf

Glanzner CH. (2014). O descompasso entre o trabalho real e o prescrito: prazer e sofrimento dos profissionais das equipes de Saúde da Família no Grupo Hospitalar Conceição. (Tese não publicada). Universidade Federal do Rio Grande do Sul. Brasil.

Lucchese, R., Castro, P., Ba, S., Rosalem, V., Silva, A., Andrade, M., Munari D., Fernandes, I., \& Neves H. (2014). Saberes profissionais na atenção primária à saúde da pessoa/família em sofrimento mental: perspectiva Le Boterf. Revista Escola de Enfermagem da USP, 48(2), 128-137. Retrieved in jun, 16, 2017 from http:// www.scielo.br/pdf/reeusp/v48nspe2/ pt_0080-6234-reeusp-48-nspe200123.pdf.

Mendes, A.M. (2007). Pesquisa em Psicodinâmica: a Clínica do Trabalho. São Paulo: Casa do Psicólogo.

Mendes, A.M. (2008). Prazer, reconhecimento e transformação do sofrimento no trabalho. ( $1^{\mathrm{a} e d}$.). Curitiba: Jurua.

Merhy, E.E., Magalhães Júnior, H.M., Rimoli, J., Franco, T.B., \& Bueno, W.S. (2007). O trabalho em saúde: olhando e experienciando o SUS o cotidiano: o debate no campo da saúde coletiva. ( $4^{\mathrm{a}}$ ed.). São Paulo: HUCITEC.

Merlo, A.R.C., Bottega, C.G., \& Perez, K.V. (2016). Saúde Mental e Trabalho no Brasil: a questão da 
atenção no Sistema Único de Saúde. Trabalho (En)Cena, 1(2), 4959. Retrieved in jun, 16, 2017 from https $/ /$ sistemas.uft.edu.br/periodicos/index .php/encena/article/view/2391/9696

Merlo, A.R.C., \& Mendes A.M.B. (2009). Perspectivas do uso da psicodinâmica do trabalho no Brasil: teoria, pesquisa e ação. Caderno de psicologia Social do Trabalho. 12(2), 141-156. Retrieved in jun, 05, 2017 from http://lpct.com.br/wpcontent/uploads/2012/11/19-

Merlo_mendes-psicodinamica-noBrasil-teoria-pesquisa-ea\%C3\%A7\%C3\%A3o.pdf

Ministério da Saúde. Conselho Nacional de Saúde. (2012). Diretrizes e Normas Regulamentadoras sobre Pesquisas envolvendo Seres Humanos. Resolução n. 466, de 12 de dezembro de 2012. Disponível em: http://conselho.saude.gov.resolucoes/ 2012/reso466.pdf

Moraes, R.D., Vasconcelos, A.C.L., Cunha, S.C.P. (2012). Prazer no Trabalho: O Lugar da Autonomia. Revista Psicologia: Organizações $e$ Trabalho, 12(2), 217-228. Retrieved in jun, 16, 2017 from http://pepsic.bvsalud.org/pdf/rpot/v1 2n2/v12n2a07.pdf

Morin, E. (2011). Introdução ao pensamento complexo. ( $4^{\mathrm{a}}$ ed.). Porto Alegre: Editora Sulina.

Patton, M.Q. (2002). Qualitative research and evaluation methods. ( $3^{\mathrm{a}} \mathrm{ed}$.). Thousand Oaks: Sage Publications.

Porcaro-Sousa, G.Z., Fukuda, C.C., \& Laros, J.A. (2015). Relação entre condições para criatividade e satisfação no trabalho de pesquisadores. Avaliação Psicológica.14(2), 169-178.

Retrieved in jun, 04, 2017 from http://pepsic.bvsalud.org/scielo.php? $\underline{\text { script }=\text { sci arttext } \& p i d=S 1677-}$ $04712015000200002 \& \operatorname{lng}=p t$.
Pires, M.R.G.M. (2011). Limites e possibilidades do trabalho do enfermeiro na estratégia saúde da família: em busca da autonomia. Revista Escola de Enfermagem da USP. 45 (2), 1710-5. Retrieved in jun, 05, 2017 from http://www.scielo.br/pdf/reeusp/v45n spe2/13.pdf

Silva, C. O., Ramminger, T. (2014). O trabalho como operador de saúde. Ciência \& Saúde Coletiva, 19(12),4751-4758 Retrieved in jun, 16, 2017 from: http://www.scielo.br/pdf/csc/v19n12/ 1413-8123-csc-19-12-04751.pdf

Silva, E.M., \& Moreira, M.C.N. (2015). Equipe de saúde: negociações e limites da autonomia, pertencimento e reconhecimento do outro. Ciência \& Saúde Coletiva. 20(10), 3033-3042. Retrieved in jun, 05, 2017 from: http://dx.doi.org/10.1590/1413812320152010.20622014.

Reibnitz, K.S., \& Prado, M.L. (2003). Criatividade e ralação pedagógica: em busca de caminhos para a formação do profissional crítico criativo. Revista Brasileira de enfermagem.56(4),439-442.

Retrieved in jun, 05, 2017 from http://www.scielo.br/pdf/reben/v56n 4/a28v56n4.pdf

Data de submissão: 22/07/2017

Data de aceite: 04/08/2017 